\title{
Prilocaine Hydrochloride
}

National Cancer Institute

\section{Source}

National Cancer Institute. Prilocaine Hydrochloride. NCI Thesaurus. Code C48012.

The hydrochloride salt form of prilocaine, an intermediate-acting local anesthetic of the amide type chemically related to lidocaine. Prilocaine hydrochloride binds to voltagegated sodium ion channels in the neuronal membrane, thereby preventing the permeability of sodium ions. This leads to a stabilization of the neuronal membrane and inhibits depolarization and results in a reversible blockage of nerve impulse generation and propagation along nerve fibres and subsequent reversible loss of sensation. 